\title{
Multi-order TODCOR: Application to observations taken with the CORALIE echelle spectrograph
}

\section{A planet in the system HD 41004 ${ }^{\star} \star \star$}

\author{
S. Zucker ${ }^{1}$, T. Mazeh ${ }^{2}$, N. C. Santos ${ }^{1,3}$, S. Udry ${ }^{1}$, and M. Mayor ${ }^{1}$ \\ 1 Observatoire de Genève, 51 ch. des Maillettes, 1290 Sauverny, Switzerland \\ e-mail: Shay.Zucker@obs.unige.ch \\ 2 School of Physics and Astronomy, Raymond and Beverly Sackler Faculty of Exact Sciences, Tel Aviv University, Tel Aviv, \\ Israel \\ 3 Centro de Astronomia e Astrofísica da Universidade de Lisboa, Observatório Astronómico de Lisboa, Tapada da Ajuda, \\ 1349-018 Lisboa, Portugal
}

Received 4 March 2004 / Accepted 17 June 2004

\begin{abstract}
In previous works we reported the detection of a brown-dwarf companion orbiting the faint stellar component of the visual binary HD 41004. This had been achieved by applying the multi-order TwO-Dimensional CORrelation (TODCOR) algorithm to CORALIE spectra. A long-term trend of the primary component had also been detected. In this paper we report follow-up observations that seem to unravel the nature of this long-term trend. The new measurements are consistent with the presence of a $2.5 M_{\mathrm{J}}$ planet in an eccentric orbit around HD $41004 \mathrm{~A}$. Thus, HD 41004 is a unique hierarchical system with a brown dwarf that accompanies the faint M-component, and a planet that orbits the brighter K-component.
\end{abstract}

Key words. methods: data analysis - techniques: radial velocities - stars: binaries: spectroscopic stars: individual: HD 41004 - stars: low-mass, brown dwarfs

\section{Introduction}

HD 41004 is a visual double system, consisting of a K1VM $2 \mathrm{~V}$ pair, with a common proper motion, a $V$-magnitude difference of 3.7 and a separation of $0.5^{\prime \prime}$ (ESA 1997). The system has been shown to exhibit periodic radial-velocity variations suggesting a planet orbiting HD $41004 \mathrm{~A}$, with an unprecedented short period of 1.3 days (Santos et al. 2002). However, Santos et al. suspected that the source of the radial-velocity variations was in fact orbital motion of the faint B-component. The low amplitude of the measured variations was the result of the faintness of the secondary spectrum.

Zucker et al. (2003) confirmed this hypothesis using the Multi-order TwO-Dimensional CORrelation algorithm. This technique allowed a separate measurement of the velocities of the two stellar components. The orbital motion of the secondary star was shown to be due to a brown dwarf in a 1.3-day orbit around HD $41004 \mathrm{~B}$, with a minimum mass of $19 M_{\mathrm{J}}$. The

^ Based on observations collected at the La Silla Observatory, ESO (Chile), with the CORALIE spectrograph at the 1.2-m Euler Swiss telescope

$\star \star$ Table 2 is only available in electronic form at the CDS via anonymous ftp to cdsarc.u-strasbrg. fr (130.79.128.5) or via http://cdsweb.u-strasbrg.fr/cgi-bin/qcat?J/A+A/426/695 primary velocities showed a clear long-term trend that called for follow-up observations to determine its nature.

Section 2 details the observations performed and the analysis that was applied to them. The results, mainly the presence of a planet around HD $41004 \mathrm{~A}$, are presented in Sect. 3, and discussed in Sect. 4.

\section{Observations and analysis}

In their preliminary analysis, Santos et al. (2002) used 86 CORALIE spectra of HD 41004, which were obtained between November 2001 and February 2002, except for one spectrum obtained in December 2000. The system was further monitored using CORALIE so that the analysis in Zucker et al. (2003) was based on 103 spectra, obtained until March 2002. In order to monitor the long-term variability of HD $41004 \mathrm{~A}$ we continued to monitor the system, adding 46 spectra, making the total 149 spectra, as of January 2004.

Following Zucker et al. (2003), the analysis used 32 CORALIE orders within the spectral range 4780-6820 , after having excluded the orders that are heavily polluted by telluric lines. Multi-order TODCOR (Zucker 2003) was used to combine the correlation functions from the 32 orders. Bluer 


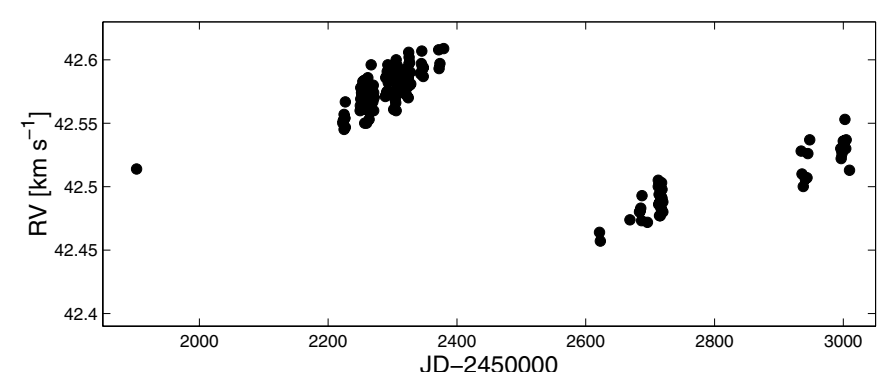

Fig. 1. Radial velocities of HD 41004 A.

orders were excluded because the secondary signal is expected to be weaker in these orders, and the instrumental response poorer (Baranne et al. 1996). Experimenting with these orders included produced a larger scatter in the derived velocities of both components, probably due to the effective lower signal-tonoise of the secondary.

The analysis used the same template spectra and light ratios that were used in Zucker et al. (2003). Thus, we used observed spectra of the K1V star HD 52698 and the M 2V star GJ 393, with a rotational broadening of $v \sin i=1 \mathrm{~km} \mathrm{~s}^{-1}$ applied to the secondary template. The stellar characteristics of these two stars are detailed in Table 2 of Zucker et al. (2003).

\section{Results}

Table 2 (available online at the CDS) lists the radial velocities we obtained. The velocities of HD41004A are shown graphically in Fig. 1. A preliminary orbital solution, based on the velocities obtained until March 2003, was presented at the 2003 Dubrovnik workshop (Zucker 2004). The solution presented at the workshop was the best of a few possible solutions, which all implied the existence of a planet candidate with a mass of about two Jupiter masses. The 17 more recent velocities allowed a better defined solution.

Examination of the velocities in Fig. 1 reveals some problems related to phase coverage. The main problem is that the periastron passage is not covered at all. This prevents a well constrained estimation of the period and the eccentricity. Furthermore, besides the first measurement, the measurements are clustered into three "clumps". Thus, one might conclude that the measurements provide only seven constraints on the solution (each "clump" providing a velocity and a slope). However, the duration of the clumps puts some constraints also on the second and higher derivatives. Thus, the orbit presented here should be considered to be a preliminary solution, prone to modification, when later measurements become available.

We chose to present here two possible orbital solutions for HD 41004 A, both shown in Fig. 2. The first one (Fig. 2, upper panel) is the best orbital solution we could find that fits the measured radial velocities, without constraining any of the orbital elements. The derived amplitude $-K-$ of this solution is accompanied by a relatively large error estimate, due mainly to the large uncertainty of the eccentricity. However, the existence of an orbital motion is clear even by a visual inspection of the data.

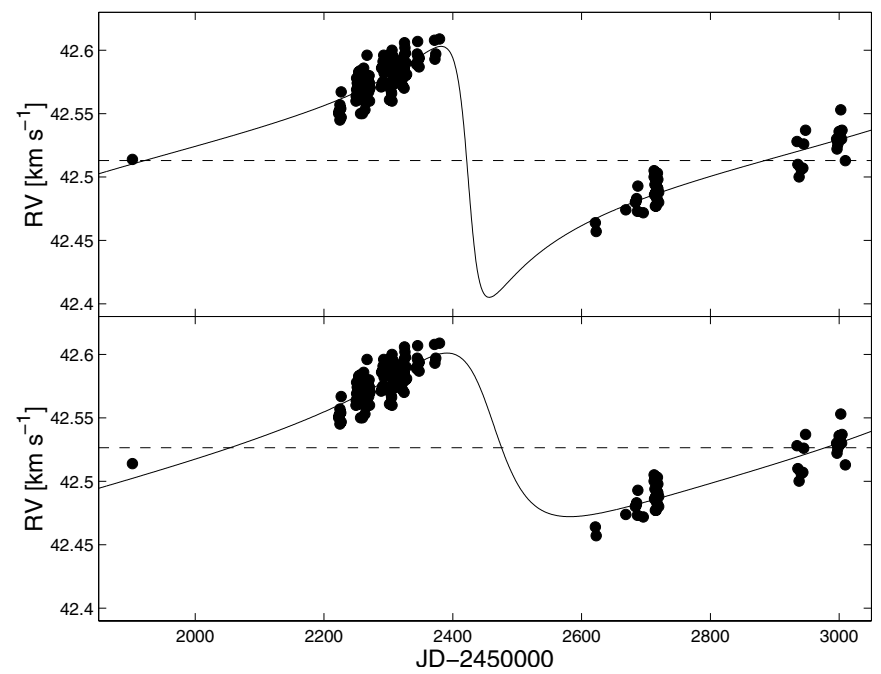

Fig. 2. Upper: radial velocities and best-fit orbital solution of HD 41004 A. Lower: radial velocities and orbital solution of HD 41004 A, with eccentricity fixed at 0.5 .

The estimated value of the eccentricity seems to be high. However, its uncertainty is also high. Therefore, for illustration purposes only, we present a second solution, where we fixed the eccentricity arbitrarily at 0.5 , demonstrating the plausibility of lower eccentricites (Fig. 2, lower panel). Fixing the eccentricity results in an artificially reduced uncertainty in the amplitude, due to a large correlation between $K$ and $e$. The residual RMS is practically unchanged, as is the estimated minimum mass.

Assuming a mass of $\sim 0.7 M_{\odot}$ for HD $41004 \mathrm{~A}$, both orbital solutions imply a companion minimum mass of about $2.5 M_{\mathrm{J}}$, well within the planetary mass range.

The orbit of HD $41004 \mathrm{~B}$, presented in Fig. 3, has not changed considerably with the addition of the new measurements, although it is now much more evident that the eccentricity is non-vanishing $-0.081 \pm 0.012$, compared to the previous value of $0.065 \pm 0.014$ in Zucker et al. (2003). The residuals may be showing a hint of a long-term trend but more measurements are needed in order to determine if it is truly significant. When a longer time baseline is available we expect to be able to measure the effect of the wide binary orbital motion.

\section{Discussion}

The orbital solution of HD $41004 \mathrm{~A}$, as presented here, is still somewhat tentative, because of the relatively poor phase coverage and the fact that only one full orbit has been monitored. Nevertheless, because of the long period, it seemed merited to publish it now, thus adding a very unique entry to the growing knowledge base about extrasolar planets. More data that will be accumulated in the future will serve to further constrain the planetary orbit.

The system HD 41004 seems to exhibit a unique hierarchical structure - a visual binary whose two stellar components are accompanied by substellar companions - a planet and a brown dwarf. The distance between A and B (at least $23 \mathrm{AU}$ ) obviously rules out dynamical instability of the brown-dwarf orbit and also of the planet orbit (e.g., Holman \& Wiegert 1999). 
Table 1. Orbital solutions of HD 41004 A and HD 41004 B.

\begin{tabular}{|c|c|c|c|c|c|c|c|}
\hline \multirow[b]{2}{*}{$P$} & \multirow[b]{2}{*}{ [days] } & \multicolumn{2}{|c|}{ HD 41004 A } & \multicolumn{2}{|c|}{$\begin{array}{l}\text { HD 41004 A } \\
\text { (e fixed) }\end{array}$} & \multicolumn{2}{|c|}{ HD 41004 B } \\
\hline & & 963 & \pm 38 & 924 & \pm 25 & 1.328300 & \pm 0.000012 \\
\hline$T$ & [JD] & 2452425 & \pm 37 & 245245 & \pm 26 & 2452434.880 & \pm 0.029 \\
\hline$e$ & & 0.74 & \pm 0.20 & 0.5 & fixed) & 0.081 & \pm 0.012 \\
\hline$\gamma$ & {$\left[\mathrm{km} \mathrm{s}^{-1}\right]$} & 42.513 & \pm 0.016 & 42.5265 & \pm 0.0049 & 41.697 & \pm 0.049 \\
\hline$\omega$ & {$\left[{ }^{\circ}\right]$} & 97 & \pm 31 & 71.7 & \pm 4.6 & 178.5 & \pm 7.8 \\
\hline$K$ & {$\left[\mathrm{~km} \mathrm{~s}^{-1}\right]$} & 0.099 & \pm 0.060 & 0.0645 & \pm 0.0027 & 6.114 & \pm 0.071 \\
\hline$a \sin i$ & {$\left[10^{-3} \mathrm{AU}\right]$} & 5.9 & \pm 1.8 & 5.5 & \pm 0.24 & 0.7440 & \pm 0.0086 \\
\hline$f(m)$ & {$\left[10^{-7} M_{\odot}\right]$} & 0.29 & \pm 0.26 & 0.257 & \pm 0.031 & 311 & \pm 11 \\
\hline$M_{2, \text { min }}^{\dagger}$ & {$\left[M_{\mathrm{J}}\right]$} & 2.54 & \pm 0.74 & 2.436 & \pm 0.098 & 18.37 & \pm 0.22 \\
\hline $\begin{array}{l}\sigma_{\mathrm{O}-\mathrm{C}} \\
N\end{array}$ & {$\left[\mathrm{~km} \mathrm{~s}^{-1}\right]$} & 0.0 & & & $\begin{array}{l}10 \\
49\end{array}$ & 0.6 & \\
\hline
\end{tabular}

${ }^{\dagger}$ Assuming $M_{\mathrm{Aa}}=0.7 M_{\odot}$ and $M_{\mathrm{Ba}}=0.4 M_{\odot}$.
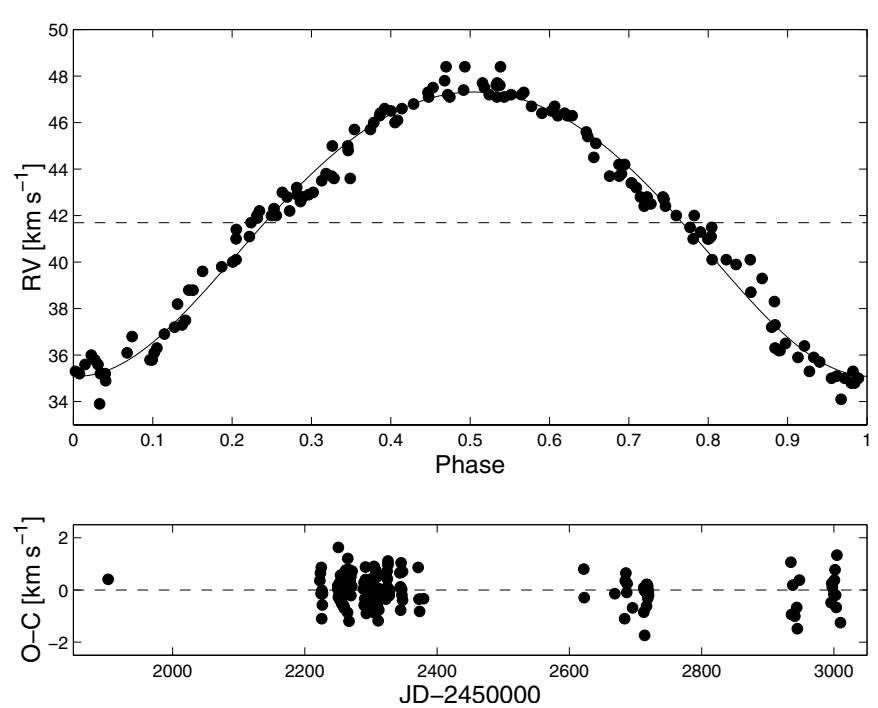

Fig. 3. Upper: phased radial velocities and orbital solution of HD 41004 B. Lower panel: the residual radial velocities after subtracting the best-fit orbit.

The eccentricity of the planet orbit is probably large, although it is currently poorly constrained. Such a high eccentricity is not unusual for orbits with periods longer than $\sim 40$ days, especially in binary systems (Eggenberger et al. 2004). The eccentricity of the brown-dwarf orbit is small but significantly non-vanishing, as was already discussed in Zucker et al. (2003), and it can be related to the interaction with the distant companion, as was shown by Mazeh (1990).

The existence of a brown dwarf companion in such a close orbit, with a period of about 1.3-day, is quite extraordinary. The term "brown dwarf desert" has been coined to represent the dearth of brown-dwarf companions in short-period orbits, and statistical proof of its existence is mounting (e.g., Halbwachs et al. 2000; Arenou et al. 2002; Halbwachs et al. 2003). The companion of HD $41004 \mathrm{~B}$ is the shortest-period brown-dwarf companion detected so far. Its presence in this under-populated region of the mass-period diagram, together with the binary nature of HD 41004, is reminiscent of the different mass-period relation for planets in single and binary stars (Zucker \& Mazeh 2002).

The system can be regarded as a hierarchical triple system, consisting of the star-brown-dwarf close pair and the distant brighter component, with a planet orbiting it. There are already two other systems with this structure - 16 Cyg (Patience et al. 2002) and HD 178911 (Zucker et al. 2002). However, in those two cases the distance between the close binary and the distant companion is much larger, of the order of hundreds of astronomical units, and the interaction details are probably completely different.

If we treat B as a single object, the system also bears some resemblance to the systems G1 86 (Els et al. 2001) and $\gamma$ Cep (Hatzes et al. 2003). In all three cases the distance between the two stellar components is about $20 \mathrm{AU}$, which is close enough to have a significant influence on the evolution of the protoplanetary disk (Artymowicz \& Lubow 1994) and the migration of the planet (Kley 2001). No planet has been detected yet in binaries with a separation smaller than $20 \mathrm{AU}$ (Eggenberger et al. 2004; Udry et al. 2003). The presence of the distant companion is not likely to affect the close orbit of the planet in Gl 86, but it may be relevant for the planets in HD 41004 and $\gamma$ Cep, whose orbits are wider than $1 \mathrm{AU}$.

Planets in multiple stellar systems have been recently shown to exhibit characteristics which seem to differ from those of planets orbiting single stars (Zucker \& Mazeh 2002; Eggenberger et al. 2004). The presence of a stellar companion to the primary star may affect the planet formation process through truncation of the protoplanetary disc (e.g., Artymowicz \& Lubow 1994) or by modifying the efficiency of planet formation process (e.g., Nelson 2000). Furthermore, it can obviously have implications regarding the long-term stability of the planetary orbit (e.g., Holman \& Wiegert 1999). Therefore, the study of planets in multiple stellar systems is expected to yield valuable information regarding the formation and evolution of planets.

The detection of the planet around HD 41004 A thus positions it in several interesting classes of objects that probe the formation and evolution of planets, brown dwarfs and binary stars. New observational programmes are currently underway 
that aim to study more deeply the characteristics of planets in binary and multiple stellar systems (e.g., Eggenberger et al. 2004; Udry et al. 2003). Hopefully, with more systems like HD 41004 we will gain a better understanding of these issues.

Acknowledgements. S.Z. wishes to acknowledge support by the European RTN "The Origin of Planetary Systems" (PLANETS, contract number HPRN-CT-2002-00308) in the form of a fellowship. T.M. gratefully acknowledges support by the Israeli Science Foundation, grant 233/03. Support from Fundação para a Ciência e Tecnologia (Portugal) to N.C.S. in the form of a scholarship is gratefully acknowledged.

\section{References}

Arenou, F., Halbwachs, J.-L., Mayor, M., \& Udry, S. 2002, EAS Publications Series, Proc. of GAIA: A European Space Project, held 14-18 May, 2001 Les Houches, France, ed. O. Bienaymé, \& C. Turon (EDP Sciences), 2, 155

Artymowicz, P., \& Lubow, S. H. 1994, ApJ, 421, 651

Baranne, A., Queloz, D., Mayor, M., et al. 1996, A\&AS, 119, 373

Eggenberger, A., Udry, S., \& Mayor, M. 2004, A\&A, 417, 353

Els, S. G., Sterzik, M. F., Marchis, F., et al. 2001, A\&A, 370, L1

ESA 1997, The Hipparcos and Tycho Catalogues, ESA SP-1200

(Noordwijk, Netherlands: ESA Publications Division)
Halbwachs, J. L., Arenou, F., Mayor, M., Udry, S., \& Queloz, D. 2000, A\&A, 355, 581

Halbwachs, J. L., Mayor, M., Udry, S., \& Arenou, F. 2003, A\&A, 397, 159

Hatzes, A. P., Cochran, W. D., Endl, M., et al. 2003, ApJ, 599, 1383

Holman, M. J., \& Wiegert, P. A. 1999, AJ, 117, 621

Kley, W. 2001, in The Formation of Binary Stars, ed. H. Zinnecker, \& R. Mathieu (San Francisco: ASP), IAU Symp., 200, 511

Mazeh, T. 1990, AJ, 99, 675

Nelson, A. F. 2000, ApJ, 537, L65

Patience, J., White, R. J., Ghez, A. M., et al. 2002, ApJ, 581, 654

Santos, N. C., Mayor, M., Naef, D., et al. 2002, A\&A, 392, 215

Udry, S., Eggenberger, A., Mayor, M., Mazeh, T., \& Zucker, S. 2003, in The Environments and Evolution of Double and Multiple Stars, IAU Coll., 191, in press

Zucker, S. 2003, MNRAS, 342, 1291

Zucker, S. 2004, in Spectroscopically and Spatially Resolving The Components of Close Binary Stars, ed. H. Hensberge, R. W. Hilditch, \& K. Pavlovski (San Francisco: ASP), in press

Zucker, S., \& Mazeh, T. 2002, ApJ, 568, L113

Zucker, S., Mazeh, T., Santos, N. C., Udry, S., \& Mayor, M. 2003, A\&A, 404, 775

Zucker, S., Naef, D., Latham, D. W., et al. 2002, ApJ, 568, 363 